\title{
MICROCHIP PATTERN RECOGNITION BASED ON OPTICAL CORRELATOR
}

\author{
Dávid SOLUS, L'uboš OVSENÍK, Ján TURÁN \\ Department of Electronics and Multimedia Communications, Faculty of Electrical Engineering and Informatics, \\ Technical University of Košice, Park Komenského 13, 04201 Košice, Slovak Republic, \\ E-mail: \{david.solus, lubos.ovsenik, jan.turan\}@tuke.sk
}

\begin{abstract}
The aim of this paper is to design the microchip pattern recognition system that is able to recognize microchip pattern based on given criteria. The inputs scenes are processed by user-friendly software created in C\# programming language and then are compared with reference pattern stored in database. Pattern recognition is based on Cambridge optical correlator, which was designed mainly for comparison and identification of images based on their similarities. The use of optical processing technology will increase the speed of processing amounts of data.
\end{abstract}

Keywords: Cambridge optical correlator, edge detection, Fourier optics, microchip pattern recognition

\section{INTRODUCTION}

Systems that offer fast and highly accurate processing results are an essential part of today. These systems include technologies based on optical data processing, which are preferred over electrical systems. Optical systems use light for processing and transmitting large amounts of data in real-time. The Cambridge optical correlator is primarily designed to compare and identify the images using optical correlation for the image comparison. The Cambridge optical correlator uses software "Fourier Optics Experimenter" (FOE), which allows users to investigate the possibilities of Fourier optics in different research areas. The disadvantage of this emerging system is its size. Many companies dealing with issues of Fourier optics are trying to miniaturize this system. Nowadays, its size shrunk by about half and it opened new possibilities for its use [1][2][3][4][5].

Goal of this paper is to present the microchip pattern recognition system (MPRS) based on optical correlator. This system is capable of recognize microchip pattern that are used in ID cards, credit cards and SIM cards.
Cambridge optical correlator is used as a comparator in this system. In our case, scanned microchip patterns are compared with reference microchip patterns stored in the database.

In Chapter two, the proposed system is presented. The particular procedure of MPRS is shown and individual processing steps are described. Chapter three describes user-friendly software of MPRS that is created in C\# programming language. Experiments and results are presented in Chapter four. Conclusion is summarized in Chapter five.

\section{MICROCHIP PATTERN RECOGNITION SYSTEM}

The microchip pattern recognitions system (Fig. 1) is designed to detect microchip from the input scene based on given criteria and then compare the pattern with reference microchip pattern from the experimental database. A program which is created in $\mathrm{C} \#$ programming language with using Aforge. NET library is used to detect the patterns.

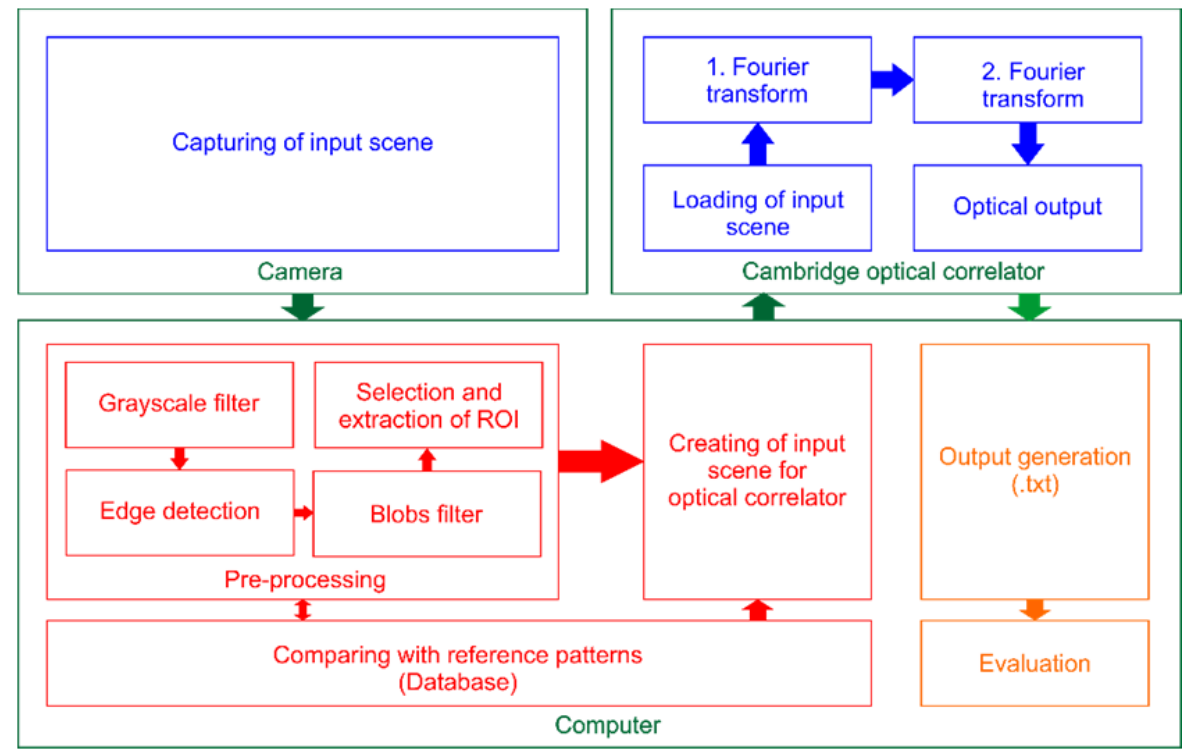

Fig. 1 Procedure of microchip pattern recognition system 


\subsection{Pre-processing}

The main task of the Pre-processing is to adjust the input image obtained by the camera for the purpose of obtaining necessary information microchip pattern. It is realized by the software for detection of the microchip pattern, which must quickly and clearly determine the region of interest (ROI). The Pre-processing consists of the following four steps:

- Grayscale filter,

- Edge detection,

- Blobs filter,

- Selection and extraction of ROI.

The first step is setting the resolution of the input image (Fig. 2a)) to $800 \times 478$ pixels, so the functions used in the program detect the microchip pattern as quickly as possible. Subsequently, Grayscale filter is applied by using the function "Grayscale()" to convert the multi-level to the grayscale image. This image carries brightness information only, no information about a colour (Fig. 2b)). Sobel detector (Fig. 2c)) was used for edge detection by using the function "SobelEdgeDetector()". This function converts image to black and white image, which get files of closed curves. These files of curves represent a range of areas and objects. After edge detection, an image contains unwanted objects or areas (blobs) that are not a part of the microchip pattern. The function "BlobsFiltering()" is applied to remove blobs that are smaller than $75 \times 75$ and bigger than $150 \times 150$ pixels (Fig. 2d)). Next step is selecting and defining the region of interest which means the place where microchip pattern might be located. The algorithm "BlobCounter()" is applied to the image - it gradually passes line by line and returns an array of rectangles that bound the field of blobs (Fig. 2e)). Last step is extraction of the selected region of interest which represents pattern (Fig. 2f)). The found microchip pattern are compared with reference microchip pattern using Cambridge optical correlator [6][7][8][9].

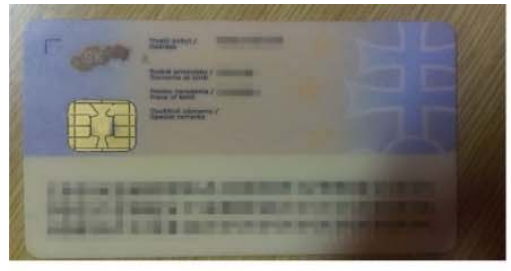

a

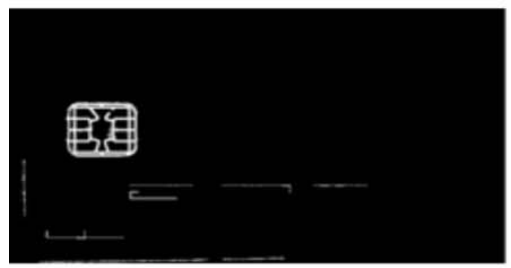

d

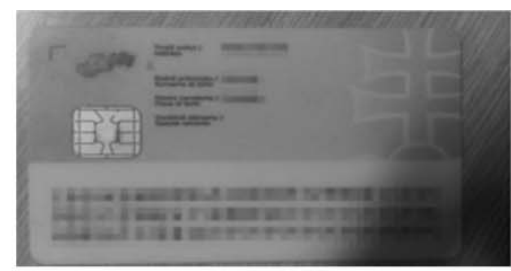

b

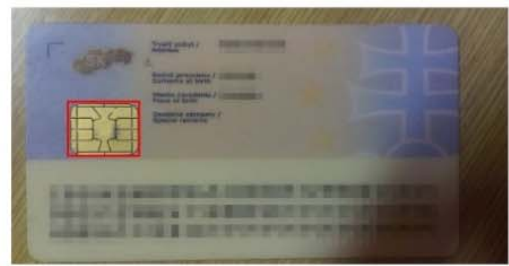

e

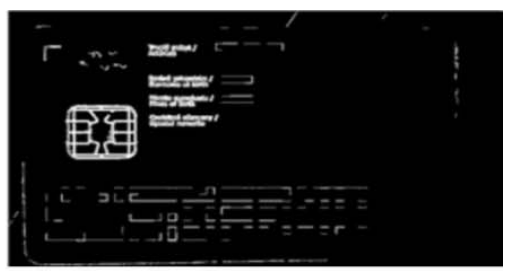

C

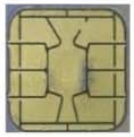

f

Fig. 2 a) Input image, b) grayscale, c) edge detection, d) blobs filter, e) selection of region of interest, f) extracted microchip pattern

\subsection{Cambridge optical correlator}

The basis of proposed system for microchips detection is the Cambridge optical correlator, whose main task is to compare the acquired image with a reference image. It is type of Joint Transform Correlator (Fig. 3), it means that optical correlation is formed by two subsequent Fourier transforms. Joint Transform Correlators are distinguished by the fact that the Fourier spectra are summed together.

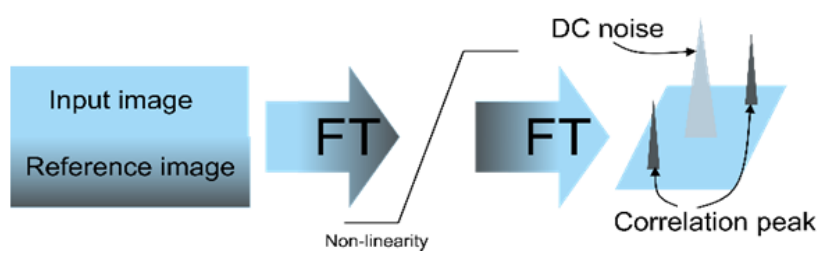

Fig. 3 Principle of Joint Transform Correlator

The input scene of optical correlator is formed by images of microchip pattern (acquired and reference) and optical output contains correlation peaks with size that reflects measure of the similarity of the compared images. The main and most important data is the size of the correlation peaks of the compared images. These data are generated by optical correlator and saved into a text document [9][10][11][12][13].

Mathematical expression of the input scene can be written as follows:

$f(x, y)=s(x, y)+r(x, y)$,

where $s(x, y)$ and $r(x, y)$ represents input and reference images shown in input plane. The input scene $f(x, y)$ is then Fourier transformed and produces $F(\alpha, \beta)$.

$\mathrm{F}(\alpha, \beta)=\mathrm{F}\{\mathrm{s}(\mathrm{x}, \mathrm{y})\}+\mathrm{F}\{\mathrm{r}(\mathrm{x}, \mathrm{y})\}=\mathrm{S}(\alpha, \beta)+\mathrm{R}(\alpha, \beta)$.

Using next equations, the square of absolute value of transformed input scene can be produced.

$\mathrm{J}(\alpha, \beta)=|\mathrm{F}(\alpha, \beta)|^{2}=|\mathrm{S}(\alpha, \beta)+\mathrm{R}(\alpha, \beta)|^{2}$ 


$$
=|\mathrm{S}(\alpha, \beta)|^{2}+|\mathrm{R}(\alpha, \beta)|^{2}+2|\mathrm{~S}(\alpha, \beta) \mathrm{R}(\alpha, \beta)|^{2} .
$$

The square of absolute value of complex function is equal to multiplication of function with its complex conjugated function and then we can write:

$$
\begin{aligned}
& \mathrm{J}(\alpha, \beta)=\mathrm{S}(\alpha, \beta) \mathrm{S}^{*}(\alpha, \beta)+\mathrm{R}(\alpha, \beta) \mathrm{R}^{*}(\alpha, \beta) \\
& +\mathrm{S}(\alpha, \beta) \mathrm{R}(\alpha, \beta)+\mathrm{R}(\alpha, \beta) \mathrm{S}^{*}(\alpha, \beta) .
\end{aligned}
$$

\section{PROGRAM IMPLEMENTATION OF MICROCHIP PATTERN RECOGNITION SYSTEM}

Experiments were done by Cambridge optical correlator and computer with FOE software. FOE is used to control the optical correlator and his source code is created by programming language $\mathrm{C \#}$, so user interface is created in Visual Studio 2015 in this language too. The user interface of the MPRS consists of two simple windows - Processing and Analysis [1][2][4].

\subsection{Processing}

The first window - Processing, is shown in the Fig. 4 This part of the user interface is used to process of input scenes and to save extracted region of interest which represents microchip pattern. The user selects the folder of input scene and folder where will be ROI saved. In addition, this window is shown input microchip pattern and bounded region of interest where microchip pattern might be located.

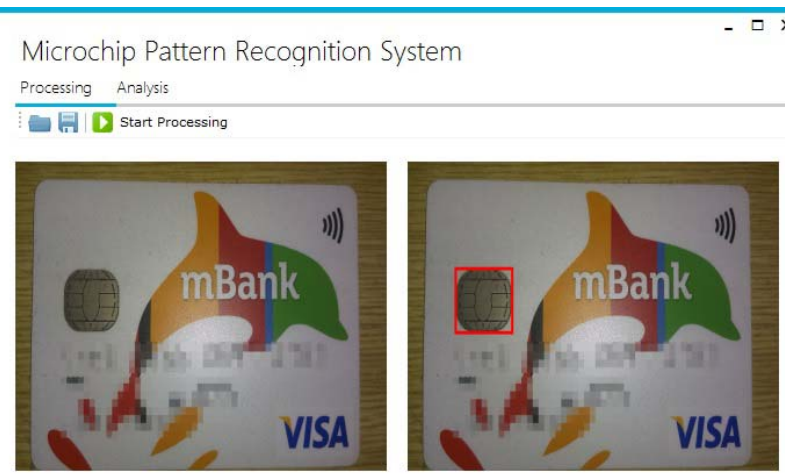

Fig. 4 Window - Processing

\subsection{Analysis}

The second window - Analysis, is shown on Fig. 5. This part of software is used to evaluation of experiments. The user selects the text file generated by Cambridge optical correlator, sets the threshold value and after that the table is created. The table contains information such as a name and image of extracted and reference microchip pattern, their intensities, an average intensity (Result).

If user pushes the button, the table of intensities will be displayed.

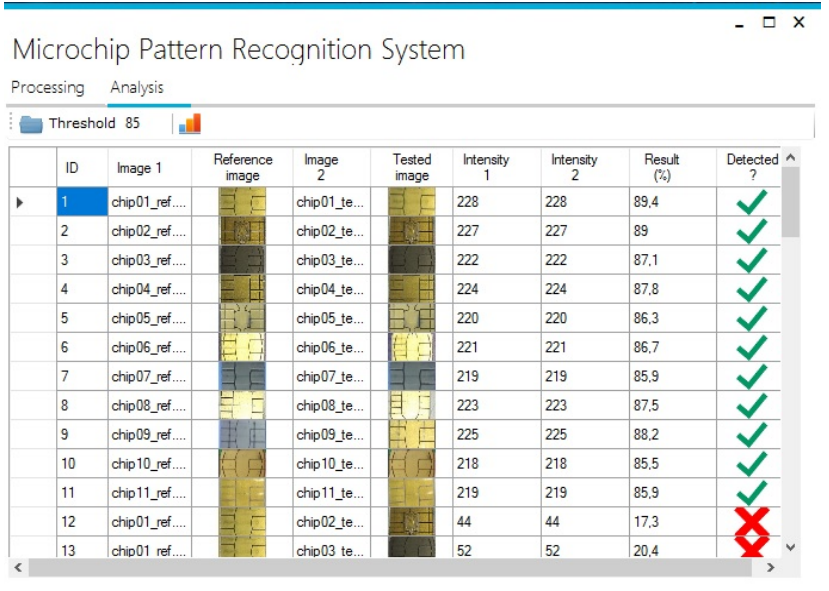

Fig. 5 Window - Analysis

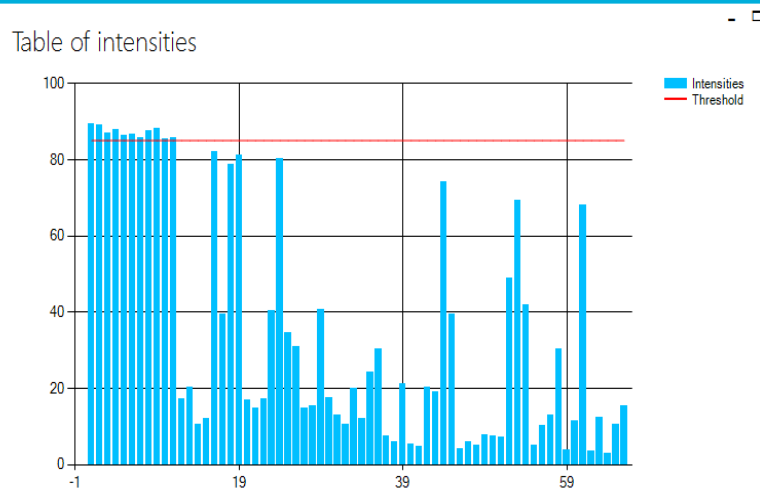

Fig. 6 Table of intensities

\section{EXPERIMENTS AND RESULTS}

The captured images of different types of microchip pattern were analysed by created MPRS based on Cambridge optical correlator. Fig. 7 is shown reference microchip pattern stored in the reference database.

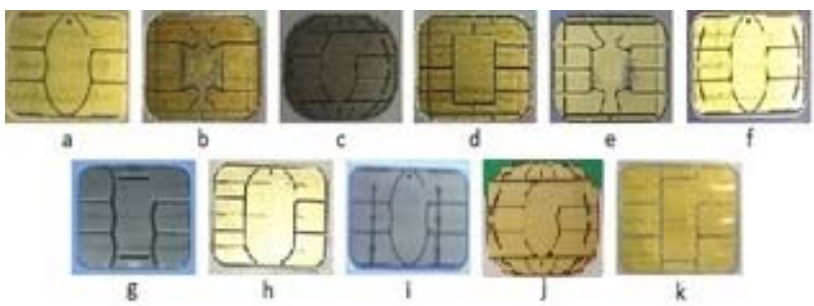

Fig. 7 Reference microchip pattern

Fig. 8 shows optical correlation between extracted and reference microchip pattern. The created input scene can be seen on Fig. 8a), Joint Power Spectrum (JPS) (Fig. $8 \mathrm{~b})$ ), JPS binary or threshold processed (Fig. 8c)) and the optical output (Fig. 8 d)) [2][4][5][10]. 


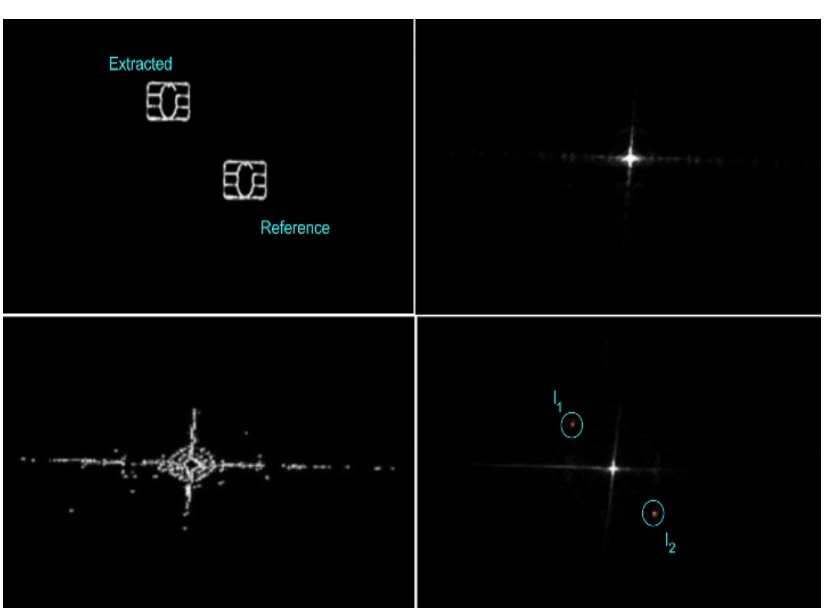

Fig. 8 Process of optical correlation between two same microchips

As it was mentioned above, the optical output contains correlation peaks and their size might be in within range $<0 ; 255>$ where value " 255 " refers to total match and value " 0 " refers to mismatch [2][3]. The equation (5) means percentage match between images situated in the input scene, where $I$ is arithmetic mean, $I_{1}$ and $I_{2}$ are intensities of the correlation peaks:

$\operatorname{Match}(\%)=\mathrm{I} / 255 * 100$,

$\mathrm{I}=\left(\mathrm{I}_{1}+\mathrm{I}_{2}\right) / 2$

The reference database contains 11 microchip patterns. 100 reference measurements were made to obtain thresholds for each of microchip patterns. Threshold can be considered as the average value of intensity of correlation peaks. The resulting thresholds are shown in the Fig. 9.

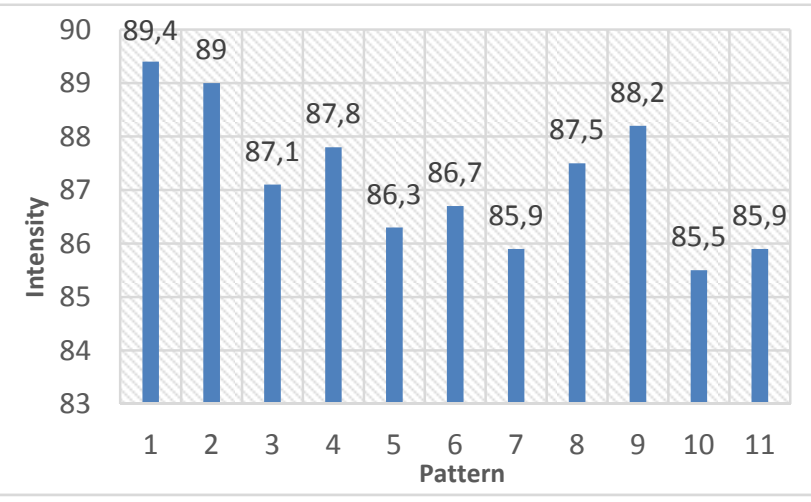

Fig. 9 Resulting intensities

It was decided that if value of percentage match is greater than $85 \%$, the extracted and reference microchip pattern are considered as the same. Values of intensities of correlation peaks of experiments shown in Fig. 8 are $I_{1}=$ 224 and $\mathrm{I}_{2}=224$. So according to equation (5) match of input images is $87,8 \%$. 66 measurements were made, in which the pattern comparison was performed. The results of percentage value of intensity of extracted and reference microchip pattern are shown in Fig. 10. As mentioned above, the maximum intensity value is 255 , e.g. exact match of compared microchip pattern. The average intensity of correlation was 89.58 what is $35.13 \%$. Some experiments had value of intensity in range $70-81 \%$ but compared images was not the same.

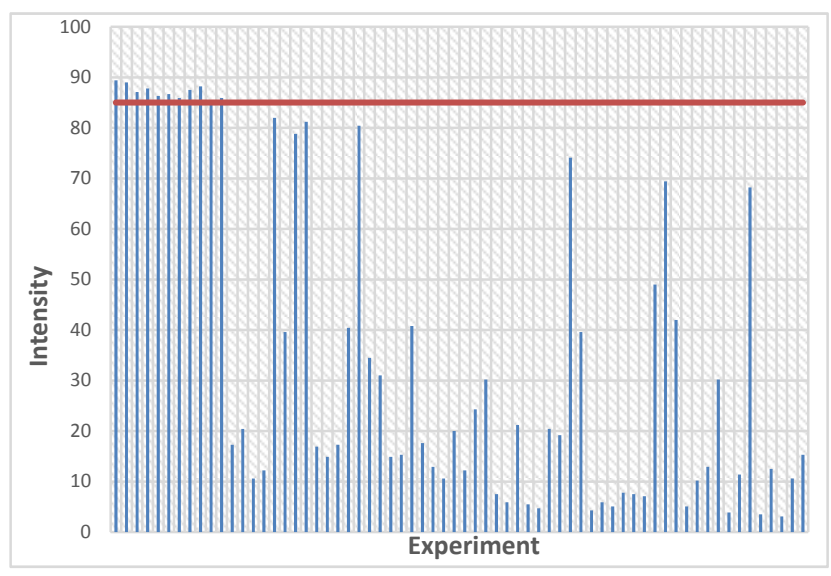

Fig. 10 Resulting intensities

\section{CONCLUSIONS}

In this paper MPRS has been described in detail. This system uses user-friendly software created in programming language $\mathrm{C} \#$ for microchip pattern extraction from static image and then these microchip patterns are compared with reference pattern stored in experimental database using Cambridge optical correlator. The average values of intensities and percentage match of compared images were obtained.

The input scene (ID cards, credit cards and SIM cards) was obtained by HD colour camera. The surface of these cards might be polluted or distorted. So, all of that had significant impact on detection and recognition.

Nowadays there are many methods of pattern recognition such a neuron networks, Support Vector Machine (SVM) or Bayesian Networks. Neural Networks and SVM are learning techniques that used model or pattern based on training data to learn and then to predict or classify data. Bayesian networks, as one of probabilistic graphical models, form an important part of artificial intelligence. They are associated with theory of probability and graph theory. In practice, they are used for creating expert systems and knowledge modelling for medical and technical applications, and references various support systems, as well as the analysis of text and image processing. These methods achieve a high percentage of recognition, such as SVM achieves results in within range $92 \%$ to $98 \%$ depending on the model. Cambridge optical correlator achieves a lower percentage of recognition but the processing speed is unmatched by other methods because the transmission medium is light [14].

\section{ACKNOWLEDGMENTS}

This publication arose thanks to the support of the Operational Programme Research and development for the project "(Centre of Information and Communication Technologies for Knowledge Systems) (ITMS code 26220120020), co-financed by the European Regional Development Fund". This work was supported by research grant KEGA no. 023TUKE-4/2017. 


\section{REFERENCES}

[1] Cambridge Correlators. Available at: http://www.cambridgecorrelators.com.

[2] Cambridge Correlators. Fourier Optics Experimenter, User Guide.

[3] ANGELSKY, O. V.: Optical Correlation Techniques and Applications, SPIE Publications, pp. 286, ISBN 978-0819465344.

[4] KUMAR, B. V. K. V. - MAHALANOBISBIS, A. JUDAY, R. D.: Correlation Pattern Recognition, Cambridge University Press, 2015.

[5] HARASTHY, T. - OVSENÍK, L. - TURÁN, J.: Current summary of the practical using of optical correlators, Acta Electrotechnica et Informatica, Vol. 12, Issue 4, 2012, pp. 30-38, ISSN 1335-8243.

[6] Digital Image Processing. Available at: http://dip.sccg.sk.

[7] AForge.NET: Computer Vision, Artificial Intelligence, Robotics. Available at: http://www.aforgenet.com

[8] GONZALES, R. - WOODS, R. - LEDDINS, S. Digital image processing using MATLAB, $2^{\text {nd }}$ ed. Natick: Gatesmark Publishing, 2009.

[9] SUMAN - PAWAN: A Survey on Various Methods of Edge Detection, International Journal of Advanced Research in Computer Science and Software Engineering, Volume 4, Issue 5, May 2014, ISSN 2277 128X.

[10] SOLUS, D.: Optical Correlator in Image and Video Processing Systems, SCYR 2015: 15th Scientific Conference of Young Researchers, May 19th, 2015, Herl'any, Slovakia, TU, 2015, pp. 96-99, ISBN 97880-553-2130-1.

[11] LAYTON, A. - MARSH, R.: Object distance detection using a joint transform correlator, IEEE International Conference on Electro Information Technology, 2016, No. 7535326, pp. 707-709, DOI: 10.1109/EIT.2016.7535326.

[12] AMBS, P.: Optical Computing, A 60-Year Adventure, Advances in Optical Technologies Journal, 2010.
[13] TÓTH, J. - OVSENÍK, L. - TURÁN, J.: Free space optics experimental system - long term measurements and analysis, Acta Electrotechnica et Informatica, Volume 15, Issue 2, 2015, pp. 26-30, ISSN 1335-8243.

[14] NISKH, P. - VARMA, M. N. - NAIK, R. R.: Principle component analysis based intrusion detection system using support vector machine, 2016 IEEE International Conference on Recent Trends in Electronics, Information \& Communication Technology (RTEICT), Bangalore, India, 2016, pp. 1344-1350.

Received January 4, 2017, accepted April 3, 2017

\section{BIOGRAPHIES}

Dávid Solus received his M.S degree from "Infoelectronics" Technical University of Košice in 2014 at Department of Electronics and Multimedia Telecommunications, Faculty of Electrical Engineering and Informatics of Technical University of Košice. Since September 2014 he has been with Technical University of Košice as $\mathrm{PhD}$. student. His research interests include Optical correlator in image and video processing systems.

Luboš Ovseník (doc., Ing., PhD.) received Ing. (MSc.) degree in radioelectronics from the Technical University of Košice in 1990. He received PhD. Degree in electronics from Technical University of Košice, Slovakia in 2002. Since February 1997, he has been at the Technical University of Košice as Associate Professor for electronics and information technology. His general research interests include optoelectronic, digital signal processing, photonics, fiber optic communications and fiber optic sensors.

Ján Turán (Dr.h.c., prof., RNDr., Ing., DrSc.) received Ing. (MSc.) degree in physical engineering with honours from the Czech Technical University, Prague, Czech Republic, in 1974, and RNDr. (MSc.) degree in experimental physics with honours from Charles University, Prague, Czech Republic, in 1980. He received a CSc. (PhD.) and DrSc. degrees in radioelectronics from Technical University of Košice, Slovakia in 1983, and 1992, respectively. Since March 1979, he has been with the Technical University of Košice as Professor for electronics and information technology. His research interests include digital signal processing and fiber optics, communication and sensing. 